Department of Economics- FEA/USP

\title{
Asset Pricing under Quantile Utility Maximization
}

\author{
Bruno Cara GiovannetTi
}

WORKING PAPER SERIES № 2012-16 


\title{
DEPARTMENT OF ECONOMICS, FEA-USP \\ WORKING PAPER № 2012-16
}

\section{Asset Pricing under Quantile Utility Maximization}

Bruno Cara Giovannetti; e-mail: bcg@usp.br

\begin{abstract}
:
"Focus on the downside, and the upside will take care of itself" is a famous quote among professional investors. By considering an agent who follows this advice, we reproduce the first and second moments of stock returns, risk-free rate and consumption growth. The agent's behavior towards risk is analogous to a relative risk aversion of about 3 under expected utility, the elasticity of intertemporal substitution is about 0.5 and the time discount factor is below 1 . In particular, the proposed model separates time and risk preferences in an innovative wav.
\end{abstract}

Keywords: asset prices, asymmetric preferences, quantile utility

JEL Codes: G11, G12 


\title{
Asset Pricing under Quantile Utility Maximization*
}

\author{
Bruno C. Giovannetti ${ }^{\dagger}$
}

June 15, 2012

\begin{abstract}
"Focus on the downside, and the upside will take care of itself" is a famous quote among professional investors. By considering an agent who follows this advice, we reproduce the first and second moments of stock returns, risk-free rate and consumption growth. The agent's behavior towards risk is analogous to a relative risk aversion of about 3 under expected utility, the elasticity of intertemporal substitution is about 0.5 and the time discount factor is below 1 . In particular, the proposed model separates time and risk preferences in an innovative way.
\end{abstract}

JEL classifications: G11, G12

Keywords: asset prices, downside risk, quantile utility

${ }^{*}$ I would like to thank Dennis Kristensen for invaluable support on this work. I also thank Andrew Ang, Pierre-André Chiappori and Marcelo Moreira for constant and crucial advice, and Ricardo Brito, John Donaldson, Guilherme Martins, Marcos Nakaguma, Walker Hanlon, Ricardo Reis, Bernard Salanié, and the participants at the Columbia Econometrics Colloquium, Applied Micro Colloquium and Finance Colloquium, and the Economics Seminars at Fundação Getúlio Vargas (EESP), Insper and Universidade de São Paulo for important comments and discussions. All errors left are only mine.

${ }^{\dagger}$ Department of Economics, University of Sao Paulo. E-mail: bcg@usp.br 
A famous quote among professional investors is "Focus on the downside, and the upside will take care of itself". In this paper, we consider a consumer-investor who follows this advice. Surprisingly, the consumption-based asset pricing model that emerges from this idea explains the main existing puzzles found within the asset pricing literature. These include the equity premium and the risk-free rate puzzles, the countercyclicality of the equity premium and the procyclicality of the risk-free rate.

In the proposed model, the consumer-investor is concerned with the so-called downside risk.This is done by replacing the standard setting of expected utility optimizing agents with the concept of quantile utility. Under this framework, the agent summarizes a risky situation using a worst-case scenario which is a function of his downside risk aversion. The more downside risk averse the agent, the worse the worst-case scenario he considers. The $\tau$ quantile of a continuous random variable can be interpreted as the worst possible outcome that can occur with probability $1-\tau$. Hence, instead of maximizing the expected value of his utility function, the agent maximizes a given $\tau$ quantile of it. As we will see, $\tau$ defines his downside risk aversion: the lower $\tau$, the higher the downside risk aversion. ${ }^{1}$

This is a novel extension of the static decision-theoretical framework developed by Manski (1988) and Rostek (2010) for a dynamic asset pricing setting. In a two-period standard economy with one risky and one risk-free asset, we can derive an arbitrage-free asset pricing model, where both main characteristics of the canonical expected utility consumption-based approach (Hansen and Singleton (1982), Mehra and Prescott (1985), hereinafter, the canonical model) are modified. The equity premium is no longer based on the covariance between the risky return and the consumption growth. Instead, it is a linear function of the risky return standard deviation. In addition, risk aversion and elasticity of intertemporal substitution (EIS), which are linked throughout a single parameter in the canonical model, are automatically disentangled in a simple way.

\footnotetext{
${ }^{1}$ One could say that the agent's objective function is given by the value at risk (VaR) of his utility. However, since $\tau$ here is a free parameter defining preference towards risk, it is not restricted to being close to zero (as in standard VaR applications).
} 
These two endogenous changes are the main drivers of the good empirical results. Since stock returns historically have a high standard deviation, the price of such a risk, i.e., the level of downside risk aversion, will not have to be high to match the empirical excess returns. Moreover, the attitude towards intertemporal substitution is not polluted by risk preferences.

To reproduce (i) the first and second moments of the risk-free return, the equity premium, and the consumption growth, (ii) the low covariance between risky return and consumption growth, (iii) the countercyclical risk premium, and (iv) the procyclical risk-free rate that we see in data, our model requires only three parameters related to preferences: a downside risk aversion $(\tau)$ of about 0.43 , an EIS $(\psi)$ of about 0.5 and a time discount factor $(\beta)$ of less than 1. A downside risk aversion of such a magnitude is reasonable in that it produces reasonable certainty equivalents for bets on continuously distributed random variables (stock indexes, for example). By comparing certainty equivalents under quantile and expected utility maximization, an agent with this level of downside risk aversion is analogous to an expected utility agent with a relative risk aversion coefficient of 3 . According to Mehra and Prescott (1985) reasonable values for such a parameter would be between 1 and 10. An EIS of about 0.5 is also an acceptable value. In a recent work using microdata, Engelhardt and Humar (2009) estimate the EIS to be 0.74 , with a $95 \%$ confidence interval that ranges from 0.37 to 1.21 . Using macrodata and separating stockholders from nonstockholders, Vissing-Jorgensen (2002) estimates the EIS around 0.4 and 0.9 for these respective groups.

To illustrate the main differences between the predictions of our framework and the predictions of the canonical model, we first derive equations in closed-form for the risky return, the risk-free rate, and the equity premium. These equations come from combining the Euler equations of the quantile agent with the standard assumption of joint lognormality of returns and consumption growth. In order to replicate the well-evidenced existence of predictability in future excess returns, we then allow for time-varying economic uncertainty in the aggregate economy dynamics. From this, a countercyclical risk premium and a procyclical risk-free rate are produced. Taking the model to data, we first perform 
simulation exercises, matching the first and second moments of consumption growth, risk-free rate and excess returns. Then, we estimate the model free of distributional assumptions using a simple two-step procedure.

Since the quantile agent summarizes a risky situation using a worst-case scenario, our model considers the fact that people care asymmetrically about good and bad outcomes. Therefore, it belongs to the class of models related to asymmetric preferences, such as Epstein and Zin (1990, 2001), Bekaert, Hodrick and Marshall (1997), Barberis, Huang and Santos (2001), Routledge and Zin (2010), and Feunou, Jahan-Parvar and Tédongap (2011).

The good empirical results from, for instance, Barberis, Huang and Santos (2001) and Routledge and Zin (2010), indicate that consideration of asymmetric preferences over good and bad outcomes is a promising path for theories on choices and, in particular, for a well-accepted resolution of the asset pricing puzzles. Nevertheless, such models have a large number of preference-related parameters, which is crucial for their success, and this is a delicate issue. ${ }^{2}$

First, it is not easy to translate the models into a comprehensive view of the whole process. Second, it is hard to assign precisely the corresponding importance of each parameter to the obtained results. Finally, and perhaps most problematic, matching data by augmenting the parametric dimension is subject to the standard over-fitting critique. According to this critique, the larger number of parameters may simply describe better the noise in the data, rather than the underlying economic relationships. In other words, these models could be providing spurious data-fitting. ${ }^{3}$

\footnotetext{
${ }^{2}$ Barberis, Huang and Santos's (2001) model has six parameters related to preference. Routledge and Zin's (2010) has five.

${ }^{3}$ This tense relationship between the augmentation of the expected utility framework with additional parameters and the over-fitting critique is raised, for instance, by Zin (2002). Based on that article, Watcher (2002) claims that "behavioral models leave room for multiple degrees of freedom in the utility function. Taken to an extreme, this approach could reduce structural modeling to a tautological, data-fitting exercise" and "I believe that parsimony lies at the root of what Zin refers to as reasonableness. A parsimonious model is a model in which the number of phenomena to be explained is much greater than the number of free parameters."
} 
The present paper helps to clarify such issues. The developed model is quite parsimonious, requiring only three preference-related parameters: the time discount factor; the EIS; and the downside risk aversion. At the same time, it solves the main asset pricing puzzles addressed by Barberis, Huang and Santos (2001) and Routledge and Zin (2010). Given that, this study makes two important contributions to the literature. Given its ability to explain the financial puzzles parsimoniously, it (i) offers a simpler view regarding the relationship between asymmetric preferences and financial data, and (ii) provides evidence that the good empirical results obtained by the studies employing asymmetric preferences are not due to over-fitting.

The rest of this work is organized as follows. Section I presents the quantile utility agent in its general form and derives some basic results of asset pricing under quantile maximization. Section II solves the model under lognormality and simulates from it. Section III discusses how to estimate the model free of distributional assumptions and presents the results. Section IV concludes.

\section{Quantile Utility Maximization and Asset Pricing}

In this section, we first present the elements of the quantile utility model, following Manski (1988) and Rostek (2010). Then, we apply this theoretical-decision framework to asset pricing.

\subsection{Quantile utility maximization elements}

A general choice theory for quantile maximizing agents was developed recently. Rostek (2010) is the first study to axiomatize the quantile utility agent. Notwithstanding, the quantile maximization model for decision making under uncertainty was first proposed 23 years ago by Manski (1988).

The main idea is simple. An agent, when facing a situation where he has to choose among uncertain alternatives, picks the one that maximizes some given quantile of the utility distribution instead of its mean, as in the expected utility model. In this framework, the agent cares about the worst outcome 
that can happen with a given probability. For instance, the given quantile can be the median of the utility distribution, or the 0.25 quantile. In the case of the 0.25 quantile for example, when evaluating an uncertain situation, he looks at the worst outcome that can occur with 75 percent probability (i.e., the chance of the realized scenario being better than the scenario he considers is 75 percent).

The quantile of concern is an intuitive measure of pessimism. If agent $A$ looks at the worst that may happen in 90 percent of the situations, i.e., quantile 0.10 , and agent $B$ looks at the worst that may happen in 60 percent of the situations, i.e., quantile 0.40 , we would naturally classify agent $B$ as more optimistic than agent $A$ : agent $A$ picks a more conservative scenario to summarize the lottery. Figure 1 illustrates this for a lottery that follows a normal distribution. As we shall see below, the quantile of concern defines also the agent's downside risk preference. Hence, downside risk preference is closely related to our standard notion of optimism-pessimism.

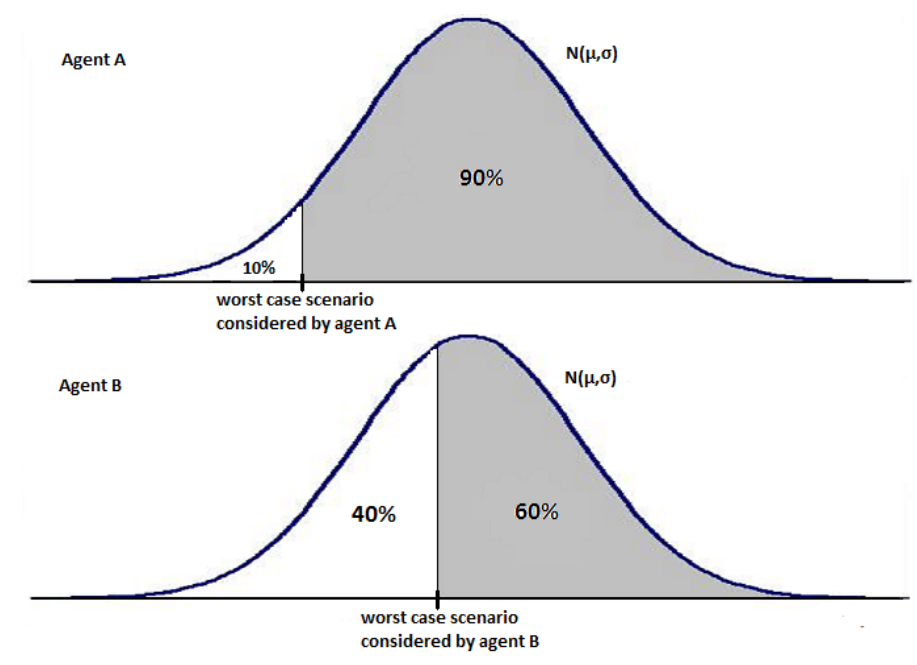

Figure 1. The quantile utility agent's reasoning.

In a more formal way, let $\mathcal{S}$ be a set of states of the world $s \in \mathcal{S}$, and $\mathcal{X}$ be an arbitrary set of payoffs $x, y \in \mathcal{X}$. Then, the agent has to choose among simple acts $h: \mathcal{S} \rightarrow \mathcal{X}$, which map from states to payoffs. Let $\mathcal{A}$ be the set of all such acts, and $E=2^{\mathcal{S}}$ be the set of all events. Define $\pi$ to be a probability measure on $E$, and $u$ a utility function over payoffs $u: \mathcal{X} \rightarrow \mathbb{R}$. For each act, $\pi$ 
induces a probability distribution over payoffs, referred to as a lottery. Given that, let $G, H$ denote the random variables (payoffs) induced by the acts $g, h \in \mathcal{A}$, respectively. Finally, define $F_{G}$ and $F_{H}$ as the lotteries induced by the acts $g$ and $h$, i.e., the cumulative distribution functions of $G$ and $H$, respectively.

A decision maker is defined as a $\tau$-quantile maximizer if there exists a unique $\tau \in[0,1]$, a probability measure $\pi$ on $E$, and a utility function $u$, such that for all $g, h \in \mathcal{A}$,

$$
g \succ h \Leftrightarrow Q^{\tau}(u(G))>Q^{\tau}(u(H)),
$$

where $Q^{\tau}(\cdot)$ represents the $\tau$-th quantile of a random variable.

As always, we can think in terms of the lotteries, that is,

$$
F_{G} \succeq F_{H} \Leftrightarrow Q^{\tau}(u(G)) \geq Q^{\tau}(u(H)) .
$$

\section{Downside risk aversion}

For the standard expected utility agent, we may understand risk preferences using the following logic. First we define riskiness. We say that the lottery $F_{H}$ is riskier than the lottery $F_{G}$ if $F_{G}$ second-order stochastic dominates ${ }^{4}$ (SSD) $F_{H}$ (see Rothschild and Stiglitz (1970)). Then, we define $\Upsilon$ to be the class of all pairs of lotteries that SSD one another, i.e., $\Upsilon=\left\{\left(F_{G}, F_{H}\right): F_{G} \operatorname{SSD} F_{H}\right\}$. It is natural to classify agent $A$ as more risk averse than agent $B$ if for all pairs of distributions in $\Upsilon$, whenever $B$ prefers a distribution which SSD the other, so does $A$. Finally, we show that this will be the case if and only if the utility function of agent $A$ is "more concave" than the utility function

\footnotetext{
${ }^{4} F_{G} \operatorname{SSD} F_{H}$ if and only if
}

$$
\int_{-\infty}^{x}\left[F_{H}(t)-F_{G}(t)\right] d t \geq 0, \text { for any } x \in \mathcal{X}
$$


of agent $B$, i.e., $u_{A}(x)=h\left(u_{B}(x)\right)$, where $h(\cdot)$ is an increasing concave function. Given that, we conclude that risk-aversion is described by the concavity of the utility function.

Manski (1988) and Rostek (2010) follow the same logic to attach the quantile maximizer's attitude toward risk to the quantile he maximizes. The central point is that riskiness is characterized in a different way, the so-called downside risk: $F_{H}$ involves more downside risk than $F_{G}$ if $F_{G}$ crosses $F_{H}$ from below. We say that lottery $F_{G}$ crosses lottery $F_{H}$ from below if there exists $x, y \in \mathcal{X}$, such that $F_{G}(y) \leq F_{H}(y)$ for all $y<x$ and $F_{G}(y) \geq F_{H}(y)$ for all $y>x$. That is, downside risk is related to the probability of bad outcomes. ${ }^{5}$

Just as above, considering the class of all pairs of lotteries with the single-crossing property, $\Phi=\left\{\left(F_{G}, F_{H}\right): F_{G}\right.$ crosses $F_{H}$ from below $\}$, we say that individual $A$ is more downside risk averse than individual $B$ if, for all pairs of distributions in $\Phi$, whenever $B$ prefers a distribution which crosses the other from below, so does $A$. Given that, we can show that agent $A$ is more downside risk averse than agent $B$ if and only if $\tau_{A}<\tau_{B}$, and then $\tau$ can be defined as the downside risk aversion parameter in the decision model: the lower $\tau$, the more downside risk averse the agent.

\section{Equivariance of quantiles to monotonic transformations and its implications}

But what role does the concavity of the utility function play under this framework? Because of the property of equivariance of quantiles to monotonic transformations, the answer to this question is "none", at least for static decision problems.

A key aspect of the quantile utility model is that static decisions are invariant to any strictly increasing transformation of the utility function. If $m: \mathbb{R} \rightarrow \mathbb{R}$ is a strictly increasing function, and $X$ is a random variable, then $^{6}$

\footnotetext{
${ }^{5}$ If $F_{G}$ and $F_{H}$ have the same mean, and $F_{H}$ has more downside risk than $F_{G}$, then $F_{H}$ has also more (second-order stochastic dominance) risk than $F_{G}$. However, under different means, this is not true.

${ }^{6}$ The intution under this result is that a strictly increasing transformation of the random variables doesn't change the order of the values of their support.
} 


$$
Q^{\tau}(m(X))=m\left(Q^{\tau}(X)\right) .
$$

Hence, for lotteries $F_{G}$ and $F_{H}$,

$$
\begin{aligned}
F_{G} \succeq F_{H} & \Leftrightarrow \quad Q^{\tau}(u(G)) \geq Q^{\tau}(u(H)) \\
& \Leftrightarrow u^{-1}\left(Q^{\tau}(u(G))\right) \geq u^{-1}\left(Q^{\tau}(u(H))\right) \\
& \Leftrightarrow Q^{\tau}(G) \geq Q^{\tau}(H),
\end{aligned}
$$

where the second line follows from the fact that $u$ is a strictly increasing function.

Given that, for static problems, the agent's decision does not depend on $u$. Manski (1988) and Rostek (2010) refer to this as a robustness property: the choice is unaffected by misspecification of the utility function.

However, the utility function is relevant in intertemporal choices. When the utility function has more than one argument, it is not possible to use the equivariance property to get rid of $u$. In particular, under time-separability, the concavity of the utility function defines the preference towards intertemporal substitution as usual. This is going to play an important role in the asset pricing model, allowing the downside risk aversion and the EIS to be disentangled. This idea is not in Manski (1988) or in Rostek (2010) and, to the best of our knowledge, is explored for the first time in the present study.

\subsection{Asset pricing}

We now apply the quantile maximization decision theory to the standard intertemporal problem of a consumer-investor agent. First, we define the consumption-investment problem and solve for the 
Euler equations that the agent must respect in equilibrium. Then we discuss the Law of One Price and the no-arbitrage condition under this framework.

Consider a two-period economy with two assets, one risky and one risk-free. Define the value of the risky asset at $t+1$ to be $X_{t+1}=P_{t+1}+D_{t+1}$, where $P_{t+1}$ is the price of the asset at $t+1$ and $D_{t+1}$ is the value of some cash flow the investor received between $t$ and $t+1$ (in the case of a stock, $D$ is the dividend). Define $X_{t+1}^{f}$ to be the value of the risk-free asset at $t+1$ and $P_{t}^{f}$ its price at $t$. Let $C_{t}$ be the agent's consumption at $t, \xi$ and $\xi^{f}$ be the quantity of the risky and risk-free assets he buys at $t$ respectively, and $W_{t}$ be his initial wealth. Then, under time-separability, he solves:

$$
\begin{aligned}
\underset{\xi, \xi^{f} \in \mathbb{R}^{2}}{\operatorname{Max}} Q_{t}^{\tau}\left(u\left(C_{t}\right)+\beta u\left(C_{t+1}\right)\right) \\
\text { s.t. } \quad C_{t}=W_{t}-P_{t} \xi-P_{t}^{f} \xi^{f} \\
\\
C_{t+1}=X_{t+1} \xi+X_{t+1}^{f} \xi^{f}
\end{aligned}
$$

where $\beta$ is the time discount factor, $u$ is the utility function, $Q_{t}^{\tau}(x)$ is the $\tau^{\text {th }}$ quantile of the conditional distribution of the random variable $x$ (conditional on the information set available at time $t$ ).

This agent derives utility only from consumption, as usual, and cares about the worst outcome (in terms of the utility for both periods) that may occur with probability $(1-\tau)$. In other words, this agent follows the famous advice "Focus on the downside, and the upside will take care of itself". As discussed in sub-section I.A, the higher his level of downside risk aversion, the lower $\tau$.

A key feature of problem (2) is that downside risk aversion and elasticity of intertemporal substitution (EIS) are automatically disentangled. This is a direct consequence of the quantile's equivariance for monotonic transformations. Note that, according to equation (1), we have

$$
\begin{aligned}
& Q_{t}^{\tau}\left(u\left(C_{t}\right)+\beta u\left(C_{t+1}\right)\right) \\
= & u\left(C_{t}\right)+\beta u\left(Q_{t}^{\tau}\left(C_{t+1}\right)\right),
\end{aligned}
$$


since $u$ is a strictly increasing function.

Hence, all uncertainty in problem (2) is resolved by parameter $\tau$, since $Q_{t}^{\tau}\left(C_{t+1}\right)$ is deterministic at $t$. The only role played by $u$ is to discount consumption across time: depending on the concavity of $u$, the agent will combine present consumption, $C_{t}$, and the certainty equivalent of future consumption (which, for the quantile maximizer, is equal to $Q_{t}^{\tau}\left(C_{t+1}\right)$ ). In other words, the concavity of $u$ will only define the EIS, denoted by $\psi$. Specializing $u(c)=\frac{c^{1-\gamma}-1}{1-\gamma}$, we have $\psi=\frac{1}{\gamma} \cdot{ }^{7}$ Note that such an assumption for the functional form of $u$ imposes no restriction on risk preference: it simply restricts the EIS to being constant.

The EIS parameter, $\psi=\frac{1}{\gamma}$, defines the degree of substitutability-complementarity between consumption today, $C_{t}$, and the certainty equivalent of consumption tomorrow, $Q_{t}^{\tau}\left(C_{t+1}\right)$. For $\psi \rightarrow 0$, $C_{t}$ and $Q_{t}^{\tau}\left(C_{t+1}\right)$ become perfect complements, and we have the agent's objective function given by

$$
U\left(C_{t}, Q_{t}^{\tau}\left(C_{t+1}\right)\right)=\min \left\{C_{t}, Q_{t}^{\tau}\left(C_{t+1}\right)\right\}
$$

At the other extreme, for $\psi \rightarrow \infty, C_{t}$ and $Q_{t}^{\tau}\left(C_{t+1}\right)$ become perfect substitutes, i.e., the agent maximizes

$$
U\left(C_{t}, Q_{t}^{\tau}\left(C_{t+1}\right)\right)=C_{t}+\beta Q_{t}^{\tau}\left(C_{t+1}\right) .
$$

For the intermediate case of $\psi=1$, we end up with the Cobb-Douglas

$$
U\left(C_{t}, Q_{t}^{\tau}\left(C_{t+1}\right)\right)=C_{t}\left(Q_{t}^{\tau}\left(C_{t+1}\right)\right)^{\beta} .
$$

${ }^{7}$ Defining $U\left(C_{t}, Q_{t}^{\tau}\left(C_{t+1}\right)\right)=\frac{C_{t}^{1-\gamma}-1}{1-\gamma}+\beta \frac{\left(Q_{t}^{\tau}\left(C_{t+1}\right)\right)^{1-\gamma}-1}{1-\gamma}$

we have that

$$
\psi \equiv-\frac{\frac{\partial U}{\partial Q_{t}^{\tau}\left(C_{t+1}\right)} / \frac{\partial U}{\partial C_{t}}}{Q_{t}^{\tau}\left(C_{t+1}\right) / C_{t}} \frac{d\left(Q_{t}^{\tau}\left(C_{t+1}\right) / C_{t}\right)}{d\left(\frac{\partial U}{\partial Q_{t}^{\tau}\left(C_{t+1}\right)} / \frac{\partial U}{\partial C_{t}}\right)}=\frac{1}{\gamma} .
$$


With respect to the time discount factor $\beta$, its role is to determine the marginal rate of substitution between $C_{t}$ and $Q_{t}^{\tau}\left(C_{t+1}\right)$. Therefore, $\psi$ defines the degree of substitutability-complementarity between $C_{t}$ and $Q_{t}^{\tau}\left(C_{t+1}\right)$, and $\beta$ parameterizes such a relation. ${ }^{8}$

As one may have already noticed, the equivalence stated in equation (3) only holds for a two-period setting, where there is only one random variable, namely, the consumption level in period $t+1$. In a three-period model, for instance, one would not be able to interchange the quantile and the utility functions as in (3), since the quantile function is not a linear operator when applied to more than one random variable. This would imply that, in principle, risk and intertemporal preferences would not be automatically disentangled for the quantile agent in a multi-period setting. Because of that, solving the model, as we do next, would be an onerous task.

Since the assumption that the investor lives only for two-periods is very strong, our model is mostly suggestive. However, it is helpful in that it provides guidelines concerning the first-order effect of downside risk. Note that the separability of time and risk preferences would continue to hold in a multi-period framework if we modeled the resolution of the uncertainty through a scenario-based reasoning. In this case, the agent would evaluate sequences of worst-case scenarios. Hence, his initial objective function would be

$$
u\left(C_{t}\right)+\beta u\left(Q_{t}^{\tau}\left(C_{t+1}\right)\right)+\beta^{2} u\left(Q_{t}^{\tau}\left(C_{t+2}\right)\right)+\ldots
$$

and time and risk preferences would be naturally disentangled. Another possible way to consider an infinite horizon setting would be to model an economy with overlapping generations of quantile agents living for two periods. Such variants of the model are worth exploring and should be object of future research. In this paper, however, we stick with the simple two-period version to keep things clear.

What are the implications of the quantile maximization asset pricing model? With the following proposition, proved in the appendix, we initiate this analysis.

\footnotetext{
${ }^{8}$ On the empirical side, we will see that both parameters are also separately identified by our estimation method.
} 
Proposition 1 Suppose a consumer-investor solves problem (2) and $u(c)=\frac{c^{1-\gamma}-1}{1-\gamma}$. Then, the Euler equations are given by

$$
\begin{gathered}
P_{t}=\beta\left(Q_{t}^{\tau}\left(\frac{C_{t+1}}{C_{t}}\right)\right)^{-\gamma} Q_{t}^{\tau}\left(X_{t+1}\right) \\
P_{t}^{f}=\beta\left(Q_{t}^{\tau}\left(\frac{C_{t+1}}{C_{t}}\right)\right)^{-\gamma} X_{t+1}^{f}
\end{gathered}
$$

The first step is to understand whether equations (4) and (5) respect the Law of One Price and the no-arbitrage condition. Then, we solve the model under the standard assumption of joint lognormality for returns and consumption growth, deriving closed-forms for the risky return, the risk-free rate and the equity premium in equilibrium.

Since we ignore transaction costs, any candidate for an equilibrium pricing system has to respect the Law of One Price: prices should be linear. That is, denoting $\Xi_{t}=\left(\xi_{t}, \xi_{t}^{f}\right)$ to be a portfolio formed at $t$, with price given by $P_{t}^{\Xi}$, the pricing system has to imply $P_{t}^{\Xi}=\xi_{t} P_{t}+\xi_{t}^{f} P_{t}^{f}$. Otherwise, $P_{t}$ and $P_{t}^{f}$ cannot be equilibrium prices because of arbitrage opportunities among the individual assets and the portfolio. Equations (4) and (5) respect this condition. Defining $\eta_{t}=\beta\left(Q_{t}^{\tau}\left(\frac{C_{t+1}}{C_{t}}\right)\right)^{-\gamma}$, we have

$$
\begin{aligned}
P_{t}^{\Xi} & =\eta_{t} Q_{t}^{\tau}\left(\xi_{t} X_{t+1}+\xi_{t}^{f} X_{t+1}^{f}\right) \\
& =\eta_{t}\left(Q_{t}^{\tau}\left(\xi_{t} X_{t+1}\right)+\xi_{t}^{f} X_{t+1}^{f}\right) \\
& =\eta_{t} Q_{t}^{\tau}\left(\xi_{t} X_{t+1}\right)+\eta_{t} \xi_{t}^{f} X_{t+1}^{f} \\
& =\eta_{t} Q_{t}^{\tau}\left(\xi_{t} X_{t+1}\right)+\eta_{t} Q_{t}^{\tau}\left(\xi_{t}^{f} X_{t+1}^{f}\right) \\
& =\xi_{t} P_{t}+\xi_{t}^{f} P_{t}^{f},
\end{aligned}
$$

where the second line follows from the quantile equivariance. Note that for a degenerate random variable $x, Q^{\tau}(x)=x$ for any $\tau \in[0,1]$, and this implies $Q_{t}^{\tau}\left(X_{t+1}^{f}\right)=X_{t+1}^{f}$.

As is well-known, a linear pricing system does not completely rule out arbitrage opportunities. Hence, we need to impose two mild conditions to end up with an arbitrage-free model. 
Proposition 2 Suppose that (i) the risky asset payoff $X_{t+1}$ is a continuous random variable and (ii) $\tau \in(0,1)$. Then, the pricing model given by equation (4) rules out arbitrage opportunities.

Both conditions of Proposition 2 (proved in the appendix) are reasonable. The continuity of the risky asset payoff comes for free for stock prices. The second condition, more subtle, rules out two well known agents in decision theory, the so-called MaxMin and MaxMax. The MaxMin agent $(\tau=0)$ summarizes a lottery by looking at the very worst case scenario that may take place (that is, the worst case scenario that may occur with probability 1$)$. On the other hand, the $\operatorname{MaxMax}(\tau=1)$ summarizes a lottery by looking at the very best case scenario that may take place (or, in other words, the worst case scenario that may occur with probability 0). Since both agents represent extreme behaviors (the extremely pessimistic and the extremely optimistic), excluding them is not a restrictive assumption.

In the next section, we solve the model under the standard assumption of joint lognormality for returns and consumption growth, deriving closed-forms for the risky return, the risk-free rate and the equity premium in equilibrium.

\section{Dynamics, Model Solution, and Simulation}

We solve the model with both constant and fluctuating economic uncertainty. Although the solution under constant economic uncertainty is enough to match both the risk-free rate and the risk premium under reasonable levels for the preference-related parameters, it does not generate a time-varying risk premium. To improve the model in this direction, we allow stochastic volatility in the economy dynamics. The model is then simulated under this richer environment.

\subsection{Dynamics 1: constant economic uncertainty}

Assume 


$$
\begin{aligned}
& g_{t+1}=\mu_{c}+\eta_{t+1}, \quad \eta_{t+1} \sim \operatorname{iid} N\left(0, \sigma_{c}\right) \\
& r_{t+1}=\mu_{r}+u_{t+1}, \quad u_{t+1} \sim \operatorname{iid} N\left(0, \sigma_{r}^{2}\right)
\end{aligned}
$$

where $g_{t+1}=\log \left(C_{t+1} / C_{t}\right), r_{t+1}=\log \left(X_{t+1} / P_{t}\right)$ and $\operatorname{Cov}\left(\eta_{t+1}, u_{t+1}\right)=\sigma_{c r}$.

Under this framework, the closed-forms for the risky return, the risk-free rate and the equity premium are given by the following proposition.

Proposition 3 If returns and consumption growth are jointly lognormally distributed, following (6), and the pricing system is given by equations (4) and (5), then

$$
\begin{aligned}
r_{t+1} & =-\log (\beta)+\gamma \mu_{c}+\Phi^{-1}(\tau)\left(\gamma \sigma_{c}-\sigma_{r}\right)+u_{t+1} \\
r_{t+1}^{f} & =-\log (\beta)+\gamma \mu_{c}+\gamma \sigma_{c} \Phi^{-1}(\tau) \\
E_{t}\left(r_{t+1}-r_{t+1}^{f}\right) & =-\sigma_{r} \Phi^{-1}(\tau)
\end{aligned}
$$

where $r_{t+1}^{f}$ refers to the risk-free asset return and $\Phi^{-1}$ is the inverse of the cumulative distribution function of a standard normal random variable.

To gain intuition on equations (8) and (9), it is useful to compare them to the analogous equations from the canonical expected utility model. As first derived by Hansen and Singleton (1983), it is well-known that under expected utility maximization and lognormality of returns and consumption growth we have

$$
r_{t+1}^{f}=-\log (\beta)+\gamma \mu_{c}-\frac{1}{2} \gamma^{2} \sigma_{c}^{2}
$$

and

$$
E_{t}\left(r_{t+1}-r_{t+1}^{f}\right)=-\frac{1}{2} \sigma_{r}^{2}+\gamma \sigma_{c r}
$$


We first focus on the predictions for the risk-free return. First, in both models, the risk-free rate is linear in expected consumption growth with the slope equal to the inverse of the elasticity of intertemporal substitution. The lower the EIS (i.e., the higher the desire for consumption smoothing across time), the higher the risk-free rate. This effect is increasing in the expected consumption growth, meaning that the agent will be less willing to save if he expects tomorrow's consumption to be higher.

Second, also common to both models, the higher the rate at which the agent discounts future utility (the lower $\beta$ ), the higher the risk-free rate he requires in order to save.

Third, and this is a first novelty of the quantile approach, a higher variability of consumption growth may have either positive or negative effects on the level of the risk-free rate under the quantile model. If $\tau>0.5$, a high standard deviation of consumption growth generates a high risk-free rate. If $\tau<0.5$, a high standard deviation of consumption growth generates a low risk-free rate. The intuition for this is clear: if the agent is optimistic $(\tau>0.5)$, a higher variability is interpreted by him as a higher chance of getting a high level of consumption tomorrow and hence, he becomes less willing to save (higher risk-free rate). In the case of pessimism $(\tau<0.5)$, a higher variability is interpreted as a higher chance of getting a low level of consumption tomorrow, which leads the agent to save more (lower risk-free rate). The strength of this effect, as expected, is increasing in the desire of smoothing consumption across time $(\gamma)$.

The separation of intertemporal and risk preferences under the quantile model becomes evident when we compare the third terms of equations (8) and (10). In equation (10), we have $\gamma^{2}$, where one $\gamma$ stands for the risk aversion and the other $\gamma$ is the inverse of the EIS. In equation (8), we have the product between the inverse of the EIS and a function of the downside risk aversion.

We now turn to the equity premium equation (9). The risk premium does not depend on the covariance between consumption and stock returns as in the canonical model but, instead, on the 
standard deviation of the stock return. ${ }^{9}$ A higher standard deviation may require either a higher or a lower expected return, depending again on whether $\tau$ is greater or less than 0.5. The intuition is the same as above: under optimism $(\tau>0.5)$, a high variability is interpreted as a high chance of getting good returns which, therefore, increases prices (decreasing expected returns). Under pessimism $(\tau<0.5)$ a high variability means a high chance of getting bad returns which causes prices to decrease (increasing expected returns).

These differences imply a better performance of the quantile model when taken to data. Because risk and time preferences are now disentangled we have degrees of freedom to fit both the risk-free rate and the equity premium (just as in Epstein and Zin (1989)). Moreover, the source of risk has now changed. Under expected utility, the covariance between consumption and risky return is the source of risk. This is empirically low, generating the necessity of a high risk aversion to match the equity premium. However, under quantile utility, risk is determined by the standard deviation of the risky return. This value is high in data and, therefore, we attenuate the role of the downside risk aversion.

Yearly US data on consumption and returns ranging from 1889 to 2009 can be found on Professor Robert Shiller's website. ${ }^{10}$ The risky and risk-free returns are from the S\&P 500 and 1-year treasury bill, respectively. The series for per capita consumption are based on the NIPA and NBER series of consumption.

According to this data set, the average real stock log return has exceeded the average treasury bills $\log$ return in about 5 percent per year in the post-war period. Stock log return has had a standard deviation about 17 percent per year, and the covariance between stock log return and per capita log consumption growth has been about 0.2 percent. Inserting these values into equation (11) and solving

\footnotetext{
${ }^{9}$ The variance term that shows up in equation (11) is simply a Jensen's inequality adjustment (since the expression is about log returns). All that matters for the difference between the risky and the risk-free returns is the covariance term.

${ }^{10}$ http://www.econ.yale.edu/ shiller/data.htm, as in November 2010.
} 
for $\gamma$, we have $\gamma=32$. Hence, in order to fit these patterns of the data, the canonical model requires a risk aversion coefficient that is too high (equity premium puzzle).

But let us suppose one is willing to accept $\gamma=32$. Then we run into the risk-free rate puzzle. The per capita log consumption growth series has presented annual mean and standard deviation of about 2.1 and 2.2 percent, respectively. The risk-free log return has been about 1.4 percent. Calibrating equation (10) with these values and solving for the time discount factor $(\beta)$, we have an absurd $\beta=1.59$ (it is unreasonable to assume that people prefer later utility).

Doing the same exercise using the quantile model equations, we first impose the left hand side of (9) to be 5 percent and the standard deviation of the risky log return to be 17 percent. Solving for $\tau$, we have $\tau=0.38$. So, in order to fit the equity premium, the agent has to care about the worst that may happen with probability 62 percent. At a first glance, this does not seem to be a high degree of pessimism. We soon will return to this point.

To compute the time discount factor $(\beta)$ necessary to fit the observable risk-free rate we should calibrate equation (8) with empirically acceptable values for the EIS. In a recent work using microdata, Engelhardt and Humar (2009) estimate the EIS to be 0.74, with a $95 \%$ confidence interval ranging from 0.37 to 1.21. By differentiating between stockholders and nonstockholders and using macrodata, Vissing-Jorgensen (2002) estimates the EIS to be around 0.4 and 0.9 , respectively. Given that, we use $\gamma=1.5$ (i.e., EIS equal to 0.67$).^{11}$

Calibrating equation (8) with $r_{t+1}^{f}=1 \%, \mu_{c}=1.9 \%, \tau=0.38, \sigma_{c}=0.021$ and $\gamma=1.5$, and solving for $\beta$, we have $\beta=1.007$, which is much better than 1.59 . By increasing $r_{t+1}^{f}$ to $2 \%$, we have $\beta=0.997$, a qualitatively acceptable value $(2 \%$ is reasonable number for the average risk-free rate as well).

\footnotetext{
${ }^{11}$ All of these estimates are obtained under the expected utility framework. Even though the EIS has nothing to do with risk, one could conjecture that if the true model is related to quantile maximization, such estimates might be biased, which would complicate the calibration of $\gamma$ under the quantile model. However, the forthcoming estimates for the EIS that I obtain under the quantile model are around these values as well.
} 


\subsection{Dynamics 2: stochastic economic uncertainty}

A limitation of the quantile model presented so far is that it does not generate a time-varying equity premium (or a time-varying risk-free rate). Because of that, the model cannot theoretically explain two well documented empirical facts: the existence of excess returns predictability and countercyclical risk premia. ${ }^{12}$ Since a significant part of the current literature on consumption-based asset pricing addresses matching time variation in expected returns, it is important to improve the quantile model in this direction.

One possible way of doing that is to incorporate fluctuating economic uncertainty into the model. Bansal and Yaron (2004) provide empirical evidence that justifies such a modification. Bansal, Khatchatrian and Yaron (2002) extensively document that a time-varying consumption volatility holds up quite well across different samples and economies. Therefore, we now assume the following dynamics for the real economy:

$$
\begin{aligned}
& g_{t+1}=\mu_{c}+\sigma_{t} \eta_{t+1} \\
& r_{t+1}=\mu_{r, t}+\varphi \sigma_{t} u_{t+1} \\
& \sigma_{t+1}^{2}=\alpha+\rho\left(\sigma_{t}^{2}-\alpha\right)+\sigma_{v} v_{t+1}
\end{aligned}
$$

where $\eta_{t+1}, v_{t+1}$ and $u_{t+1}$ are now standard gaussian random variables and $\operatorname{Cov}\left(\eta_{t+1}, u_{t+1}\right)=\sigma_{c r}$.

The stochastic volatility fluctuates around $\alpha$, and $\rho$ represents how quickly it gets pulled toward its mean. The evidence in Bansal and Yaron (2004) and Bansal, Khatchatrian and Yaron (2002) are of slow-moving fluctuations in economic uncertainty, implying a $\rho$ close to one. The conditional variances of consumption growth and return are now given by $\sigma_{t}^{2}$ and $\varphi^{2} \sigma_{t}^{2}$, respectively, and the conditional covariance between consumption growth and return is now $\varphi \sigma_{t}^{2} \sigma_{c r}$.

\footnotetext{
${ }^{12}$ See Fama and French (1989), Ludvigson and Ng (2007) and Cooper and Priestley (2009), for instance, on the countercyclicality of the risk premium.
} 
Solving for $\mu_{r, t}$, the next proposition shows that returns and risk premium are now time-variant.

Proposition 4 Under the dynamics defined in equations (12), (13) and (14) and the Euler equations

(4) and (5) we have:

$$
\begin{aligned}
r_{t+1} & =-\ln \beta+\gamma \mu_{c}+(\gamma-\varphi) \sigma_{t} \Phi^{-1}(\tau)+\varphi \sigma_{t} u_{t+1} \\
r_{t+1}^{f} & =-\ln \beta+\gamma \mu_{c}+\gamma \sigma_{t} \Phi^{-1}(\tau) \\
E_{t}\left(r_{t+1}-r_{t+1}^{f}\right) & =-\varphi \sigma_{t} \Phi^{-1}(\tau)
\end{aligned}
$$

If $\tau<0.5$ (the pessimistic agent, as discussed in the previous subsection), periods with higher economic uncertainty are periods with higher demand for saving, and hence, lower risk-free rate. This effect is increasing in the desire for consumption smoothing $\gamma$, the inverse of the EIS. Moreover, more economic uncertainty raises the risk premium, and this effect is increasing in $\varphi$ - the parameter that links economic uncertainty to return uncertainty. Therefore, the time-variation goes in the (theoretically-) intuitive direction.

As Bansal and Yaron (2004) claim, consumption and market volatilities are high during recessions. Given that, the risk premium in equation (17) is countercyclical. ${ }^{13}$ In addition, equation (16) implies a procyclical risk-free rate, in line with data as well.

\section{Simulation}

We now simulate from this model to better visualize its asset pricing implications. We simulate first the economic uncertainty from equation (14) and then feed equations (12), (15) and (16) with this series. As in Campbell and Cochrane (1999), Barberis, Huang and Santos (2001), Bansal and Yaron (2004), Bansal, Kiku and Yaron (2009) and many others, we assume that the decision interval of the agent is monthly but the targeted data to match are annual. Therefore, we simulate at the monthly frequency and aggregate to annual data.

\footnotetext{
${ }^{13}$ The counter-cyclical feature of the risk premium in the long-run risk model of Bansal and Yaron (2004) also comes from the presence of the stochastic volatility in the risk-premium equation.
} 
The stochastic volatility structure added to the model is identical to the one considered in Bansal and Yaron (2004) and Bansal, Kiku and Yaron (2009), and we calibrate parameters $\left(\alpha, \rho, \sigma_{v}\right)$ with the same values of this last paper. ${ }^{14}$ With respect to $\left(\mu_{c}, \sigma_{c r}\right)$, they are set in accordance the sample mean of the consumption growth and the sample covariance between consumption growth and risky return, respectively.

Given such values, we choose the free parameters $(\varphi, \beta, \tau, \gamma)$ seeking to match the first and second moments of the risk-free rate and excess return, and the second moment of consumption growth. Table 1 summarizes the parameters' optimal choices.

\begin{tabular}{l|c}
\hline \multicolumn{1}{c}{ parameters for monthly simulation } & value \\
\hline$\alpha$ (mean of economic uncertainty) & $0.0072^{2}$ \\
$\sigma_{\mathrm{v}}$ (standard deviation of log economic uncertainty) & $0.28 \times 10^{-5}$ \\
$\rho$ (log economic uncertainty persistence) & 0.999 \\
$\mu_{\mathrm{c}}$ (mean consumption log growth) & 0.0018 \\
$\sigma_{\mathrm{cr}}$ (covariance between $\eta$ and $\left.\mathrm{u}\right)$ & 0.5 \\
$\varphi$ (adjustment of the log return standard deviation) & 5.5 \\
$\beta$ (discount factor) & 0.9998 \\
EIS (inverse of $\gamma$ ) & 0.6 \\
$\tau$ (downside risk aversion) & 0.45 \\
\hline
\end{tabular}

Table 1. Configuration of the model parameters.

The preference-related parameters $(\beta, \tau, \gamma)$ are close to those from the previous sub-section. The time discount factor $(\beta)$ is slightly below one, the EIS of 0.6 implies $\gamma=1.66$, and the downside risk aversion is now even smaller with $\tau=0.45 .{ }^{15}$

Table 2 presents the impacts on the simulated moments of varying both the risk aversion and EIS.

\footnotetext{
${ }^{14}$ Equation (14) produces a small number (about 5\%) of negative values for $\sigma_{t}^{2}$, as in Bansal and Yaron (2004) and Bansal, Kiku and Yaron (2009). Following them, I replace these negative values with the smallest positive value generated for $\sigma_{t}^{2}$. Obviously, one could model $\log \left(\sigma_{t}^{2}\right)$ to get rid of this technical problem (but, in this case, it wouldn't be possible to follow their calibration).

${ }^{15}$ Importantly, the quantile model does not need an EIS greater than one to produce good empirical results. This is relevant when compared to Bansal and Yaron (2004). For them, it is crucial for the good results to employ an EIS greater than one, more precisely, equal to 1.5 (and this value is not empirically reasonable, as discussed before.)
} 
The other parameters are kept fixed in accordance with Table 1.

\begin{tabular}{|c|c|c|c|c|c|c|c|c|}
\hline$\tau$ & EIS & $E(r-r f)$ & $\sigma(r)$ & $E(r f)$ & $\sigma(\mathrm{rf})$ & $\mathrm{E}(\mathrm{g})$ & $\sigma(\mathrm{g})$ & $\operatorname{cov}(g, r)$ \\
\hline 0.41 & 0.1 & 10.0 & 15.8 & 3.5 & 11.2 & 2.1 & 2.7 & 0.2 \\
\hline 0.41 & 0.6 & 10.0 & 15.6 & 0.8 & 1.9 & 2.1 & 2.7 & 0.2 \\
\hline 0.41 & 1.1 & 10.0 & 15.9 & 0.5 & 1.0 & 2.1 & 2.7 & 0.2 \\
\hline 0.45 & 0.1 & 5.5 & 15.3 & 11.7 & 6.2 & 2.1 & 2.7 & 0.2 \\
\hline 0.45 & 0.6 & 5.5 & 15.3 & 2.1 & 1.1 & 2.1 & 2.7 & 0.2 \\
\hline 0.45 & 1.1 & 5.5 & 15.3 & 1.2 & 0.6 & 2.1 & 2.7 & 0.2 \\
\hline 0.49 & 0.1 & 1.0 & 15.0 & 19.8 & 1.2 & 2.1 & 2.7 & 0.2 \\
\hline 0.49 & 0.6 & 1.0 & 15.0 & 3.5 & 0.2 & 2.1 & 2.7 & 0.2 \\
\hline 0.49 & 1.1 & 1.0 & 15.0 & 2.0 & 0.1 & 2.1 & 2.7 & 0.2 \\
\hline \multicolumn{2}{|c|}{ data } & $\begin{array}{c}4.8 \\
(1.5)\end{array}$ & $\begin{array}{l}16.8 \\
(1.8)\end{array}$ & $\begin{array}{c}1.4 \\
(0.5)\end{array}$ & $\begin{array}{c}1.7 \\
(0.3)\end{array}$ & $\begin{array}{c}2.1 \\
(0.3)\end{array}$ & $\begin{array}{c}2.2 \\
(0.5)\end{array}$ & $\begin{array}{c}0.2 \\
(0.0)\end{array}$ \\
\hline
\end{tabular}

Table 2. Varying EIS and downside risk aversion (in \%).

From Table 2 we see three effects: (i) higher values of downside risk aversion (i.e., lower values of $\tau$ ) increase the mean excess return; (ii) lower values for EIS increase the mean risk-risk free return and its volatility; and, (iii) decreasing $\tau$ also impacts the mean and standard deviation of the risk-free rate, decreasing the former and increasing the latter.

The theoretical reasons for the effects related to the first moments are the same as those under constant economic uncertainty. A higher downside risk aversion implies a higher price for the risk, and therefore, a higher risk premium, justifying effect (i). A higher complementarity between consumption at $t$ and the certainty equivalent of consumption at $t+1$ implies a higher desire to smooth consumption in time, and therefore, a higher risk-free rate to justify savings from $t$ to $t+1$, which explains effect (ii). Finally, a higher downside risk aversion leads to more savings from period $t$ to period $t+1$ for a given level of economic uncertainty at $t$, lowering the risk-free rate and justifying (iii).

With respect to the effects related to the second moment of the risk-free rate, the theoretical explanations are the following. The effect in (ii) comes from the natural fact that the volatility of the risk-free rate is a function of the volatility of the economic uncertainty which is decreasing in the EIS (see equation (16)). This makes theoretical sense, since savings should respond more to economic uncertainty, the more the agent cares about smoothing consumption. The reasoning supporting the 
effect in (iii) follows the same line: the more downside risk averse the agent, the more savings should respond to economic uncertainty.

We therefore conclude that the quantile asset pricing model's predictions are theoretically solid. In addition, when calibrated with empirically reasonable parameters and $\tau=0.45$, the model is able to reproduce important patterns of financial and macroeconomic data. At this point, a natural question is: how reasonable is $\tau=0.45$ ?

\subsection{What is a reasonable value for $\tau$ ?}

Is $\tau=0.45$ more reasonable than $\gamma=32$ (the value obtained in sub-section 2.1 for the risk aversion under expected utility and lognormality) in terms of the implied attitude towards risk? Or, what is a reasonable range for $\tau$ ?

One way to evaluate $\tau$ is to compare the certainty equivalent implicit in a quantile model to the one implicit in a power utility model for risky situations with payoffs following continuous distributions, in accordance with Proposition 2.

Using certainty equivalents of simple bets to relate parameters from different models of behavior towards risk is a standard procedure in this literature. For instance, Epstein and Zin (1990) use such a strategy to compare the risk aversion levels in Yaari preferences with the risk aversion levels in the expected utility preferences (see their Tables 1 and 2). Bonomo and Garcia (1993), Epstein and Zin (2001), Routledge and Zin (2010), among others, do the same.

A simple and natural risky situation to use is the following. Suppose the agent wants to invest $\$ 1000$ and the investment return follows the same distribution considered in (6). Therefore,

$$
\ln \left(X_{t+1}\right) \sim N\left(\mu_{r}+\ln (1000), \sigma_{r}^{2}\right),
$$

where, as usual, $X_{t+1}$ is the value of the investment at $t+1$. 
For a one-year investment, the sample estimates for $\mu_{r}$ and $\sigma_{r}^{2}$ are about 0.08 and 0.03 respectively. The initial investment value is immaterial for the forthcoming conclusions.

We can first ask: what are the certainty equivalents for a quantile agent with $\tau=0.45$ and for an expected power utility agent with $\gamma=35$ for this uncertain outcome $X_{t+1}$ ?

For an expected utility agent with power utility, the certainty equivalent of a lottery with payoff $x$ is given by

$$
C E_{E U}=\left[E\left(x^{1-\gamma}\right)\right]^{\frac{1}{1-\gamma}}
$$

For a $\tau$-quantile utility agent, the value of a lottery with payoff $x$ is equal to $Q^{\tau}[u(x)]$. So, the certainty equivalent of such a lottery is the solution of $u\left(C E_{Q U}\right)=Q^{\tau}[u(x)]$. By quantile equivariance,

$$
C E_{Q U}=Q^{\tau}(x)
$$

Figure 2 presents the histogram of the uncertain investment value at $t+1$, which has mean and standard deviation around $\$ 1103$ and $\$ 212$, respectively. The vertical dashed lines are the certainty equivalents for the power utility agent with $\gamma=35$ and for the quantile agent with $\tau=0.45$ (they are around $\$ 643$ and $\$ 1057$, respectively). 


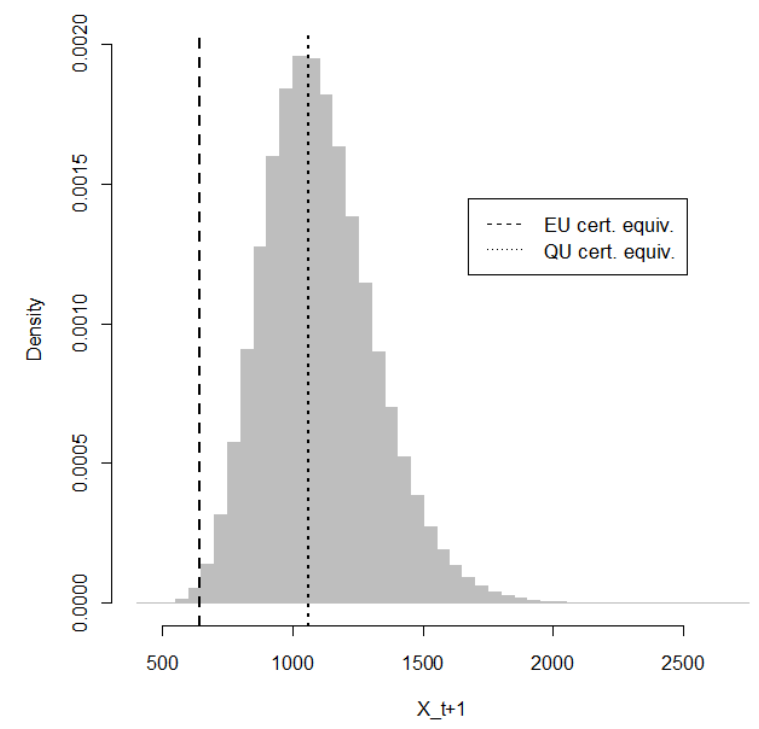

Figure 2. Histogram of uncertain payoff and certainty equivalents for $\gamma=35$ and $\tau=0.45$.

A casual review of this figure suggests that the certainty equivalent of a power utility agent with $\gamma=35$ is too small compared to what one would expect as reasonable. On the other hand, for a quantile agent with $\tau=0.45$, his certainty equivalent looks much better. However, it is already wellknown in the literature that $\gamma=35$ generates extreme outcomes in an expected utility setting. So, one can argue that basically any alternative utility specification is going to behave more reasonably. Considering that, perhaps a clearer, more illustrative way to proceed would be to ask: which value of $\gamma$ would give the certainty equivalent obtained with $\tau=0.45$ ? The answer is $\gamma=2.5$. In other words, in terms of certainty equivalents, a quantile utility agent with $\tau=0.45$ would be analogous to an expected utility agent with $\gamma=2.5$, a value which is commonly referred to as reasonable in the literature.

Pursuing this idea further, we can relate many values of $\tau$ to many values of $\gamma$ in terms of producing the same certainty equivalent for the bet defined above. Figure 3 presents this relationship. 


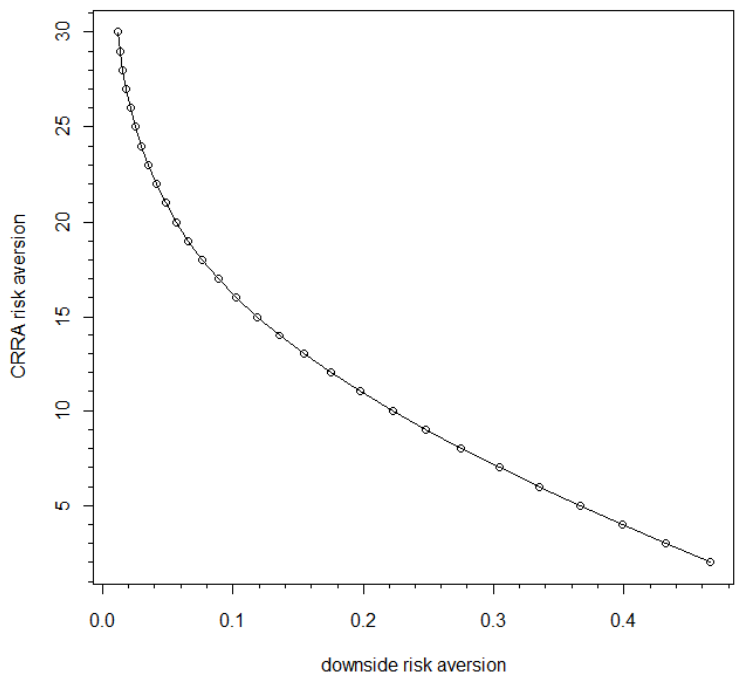

Figure 3. Values of $\tau$ and $\gamma$ that produce the same certainty equivalent in the bet defined above.

Mehra and Prescott (1985) argue that acceptable values for $\gamma$ would be between 1 and 10. Hence, for the risky situation considered, the analogous interval for $\tau$ would be $[0.22,0.48]$.

\subsection{Comparing results}

So far we have compared our results only to those from the canonical model. This was done to illustrate the new features of the present approach with respect to the predictions for the risk-free rate and the equity premium.

In this sub-section we briefly compare the results obtained to those of Epstein and Zin (1989) and Weil (1989) (three parameters), Bonomo and Garcia (1993) (four parameters) and Routledge and Zin (2010) (five parameters), and Barberis, Huang and Santos (2001) (six parameters).

By using recursive preferences, Epstein and Zin (1989) and Weil (1989) disentangle risk aversion and EIS and still have the time discount rate - the same parameters we have here. By doing so, they are able to fit both the equity premium and the risk-free rate. However, the extremely high risk aversion remains crucial. As Table 1 in Weil (1989) shows, in order to match the average of risk-free and excess returns, risk aversion and EIS have to be set at 45 and 0.1, respectively. If risk aversion 
is decreased to 1 , the premium is as low as 0.45 percent, while the mean risk-free rate reaches 25 percent. Furthermore, nothing is said about second moments.

With one extra parameter compared to our model (the one that regulates the disappointment aversion), the model in Bonomo and Garcia (1993) under a joint random walk for consumption and dividend growth rates ${ }^{16}$ produces an average equity premium on the order of 2.5 percent with standard deviation about 12.8 percent. The risk-free rate averages about 4.5 percent. This is the best they are able to get using what they consider reasonable values for their parameters.

By adding one more parameter to the disappointment aversion model, Routledge and Zin (2010) are able to generate good results with this framework. By means of a countercyclical risk aversion (produced by an endogenous variation in the probability of disappointment), they produce a large equity premium (about 6 percent) and a risk-free rate with low volatility and mean. However, they still have difficulty with fitting the risky return volatility and maintaining the 6 percent equity premium at the same time.

Barberis, Huang and Santos (2001) assume a functional form for preferences based on prospect theory, which has 6 parameters. Their model succeeds in explaining the first and second moments of the risk-free rate, the equity premium and the consumption growth, and produces a time-varying risk premium (that comes from the impact of the agent's past portfolio result on his sensitivity for future losses).

\section{Model Estimation}

The previous section presented the quantile utility asset pricing model under the assumption of joint conditional lognormality of asset returns and consumption growth. This was useful for building intuition with respect to the model. However, it is well-known that the lognormality assumption is

\footnotetext{
${ }^{16}$ Comparable to the dynamics I use here.
} 
not consistent with all the properties of historical stock returns. For example, stock log returns show weak evidence of skewness and strong evidence of excess kurtosis, at least for short horizons. Hence, it is important to understand how the model performs if we relax the lognormality assumption.

Let the vector $\theta_{0}=\left(\tau_{0}, \beta_{0}, \psi_{0}\right)$ represent the populational values for the downside risk aversion, the time discount factor and the EIS, respectively. Define $Y_{t+1}=\left(\frac{C_{t+1}}{C_{t}}, R_{t+1}, R_{t+1}^{f}\right)$ and let $Y \equiv\left\{Y_{t}: \Omega \longrightarrow \mathbb{R}_{+} \times \mathbb{R}, t=1, \ldots, T\right\}$ be a stochastic process defined on a complete probability space $(\Omega, \mathcal{F}, P)$, where $\mathcal{F} \equiv\left\{\mathcal{F}_{t}: t=1, \ldots, T\right\}$ and $\mathcal{F}_{t} \equiv \sigma\left\{Y_{s}: s \leq t\right\}$

From Proposition 1, the risky and risk-free returns in equilibrium should respect the following two equations

$$
\begin{aligned}
\beta_{0}\left(Q^{\tau_{0}}\left(\frac{C_{t+1}}{C_{t}} \mid \mathcal{F}_{t}\right)\right)^{-1 / \psi_{0}} Q^{\tau_{0}}\left(R_{t+1} \mid \mathcal{F}_{t}\right) & =1 \\
\beta_{0}\left(Q^{\tau_{0}}\left(\frac{C_{t+1}}{C_{t}} \mid \mathcal{F}_{t}\right)\right)^{-1 / \psi_{0}} R_{t+1}^{f} & =1
\end{aligned}
$$

where we now use the EIS parameter $\psi_{0}$ instead of its inverse $\gamma_{0}$.

By dividing equation (18) with equation (19) we get

$$
Q^{\tau_{0}}\left(R_{t+1} \mid \mathcal{F}_{t}\right)=R_{t+1}^{f}
$$

Rearranging equation (19), we have

$$
Q^{\tau_{0}}\left(\frac{C_{t+1}}{C_{t}} \mid \mathcal{F}_{t}\right)=\left(\beta_{0} R_{t+1}^{f}\right)^{\psi_{0}}
$$

A two-step estimation procedure

The parameter vector $\theta_{0}$ can be consistently estimated in a very simple manner, using a twostep procedure. This discussion builds intuition into the model and provides a rapid technology for 
estimating, for instance, the EIS (the estimation of the EIS under Epstein and Zin (1989) preferences, the alternative technology of disentangling risk and time preferences, is much more involving).

In a first step, we estimate $\tau_{0}$. Equation (20) implies

$$
E\left[\tau_{0}-1\left[R_{t+1}<R_{t+1}^{f}\right]\right]=0
$$

Hence, a consistent estimator of $\tau_{0}$ is

$$
\widetilde{\tau}=\frac{1}{T} \sum_{t=1}^{T} 1\left[R_{t+1}<R_{t+1}^{f}\right],
$$

which is the relative number of observations in the sample such that $R_{t+1}<R_{t+1}^{f}$. From standard arguments, its asymptotic distribution is given by

$$
\sqrt{T}\left(\widetilde{\tau}-\tau_{0}\right) \stackrel{d}{\rightarrow} N\left(0, \tau_{0}\left(1-\tau_{0}\right)\right)
$$

The intuition of equation (22) is clear. A risk-free rate of return which is frequently above the risky rate is only justifiable if the agent has a high $\tau$, that is, a low level of downside risk aversion. In this case, given the high optimism of the agent, he still invests in the risky asset in equilibrium.

Given $\widetilde{\tau}$, we can now estimate $\left(\beta_{0}, \psi_{0}\right)$ by a standard linear quantile regression. This is the case since, by the equivariance property of quantiles, equation (21) implies

$$
Q^{\tau_{0}}\left(g_{t+1} \mid \mathcal{F}_{t}\right)=\lambda_{0}+\psi_{0} r_{t+1}^{f},
$$

where $g_{t+1}=\log \left(C_{t+1} / C_{t}\right), r_{t+1}^{f}=\log \left(R_{t+1}^{f}\right)$ and $\lambda_{0}=\psi_{0} \log \left(\beta_{0}\right)$.

The only drawback of using $\widetilde{\tau}$ instead of $\tau_{0}$ in equation (23) is the usual problem with standard errors of the second step. As is well-known, they have to be corrected because of the noise produced in the first-step estimation. However, in practice, this implies no additional computational cost for our two-step procedure. In standard quantile regressions, the coefficients' asymptotic variance contains the 
unknown conditional distribution of the error term. Because of that it is common to compute standard errors by bootstrap. Hence, to address the two-step estimation issue, it is natural to incorporate the first step in the bootstrap procedure. ${ }^{17}$

From $(\widehat{\lambda}, \widehat{\psi})$ one consistently computes $\widehat{\beta}=\exp (\widehat{\lambda} / \widehat{\psi})$. The standard error of $\widehat{\beta}$ should be computed from the bootstrapped covariance matrix of $(\widehat{\lambda}, \widehat{\psi})$ by the delta method. Accordingly,

$$
\sqrt{T}\left(\widehat{\beta}-\beta_{0}\right) \stackrel{d}{\rightarrow} N\left(0, \exp \left(2 \frac{\lambda_{0}}{\psi_{0}}\right)\left(\frac{1}{\psi_{0}^{2}} \sigma_{\lambda}^{2}+\frac{\lambda_{0}^{2}}{\psi_{0}^{4}} \sigma_{\psi}^{2}-2 \frac{\lambda_{0}}{\psi_{0}^{3}} \sigma_{\lambda \psi}\right)\right),
$$

where $\sigma_{\lambda}^{2}$ is the asymptotic variance of $\widehat{\lambda}, \sigma_{\psi}^{2}$ is the asymptotic variance of $\widehat{\psi}$, and $\sigma_{\lambda \psi}$ is the asymptotic covariance between both estimators.

\section{Estimation results}

We apply the estimation procedure described above to a monthly data set. Such data frequency is used to maintain the assumption that the decision interval of the agent is monthly, as in the simulation exercise. Per capita consumption is the sum of personal consumption expenditures on services (PCES, St. Louis Fed) and personal consumption expenditures on nondurable goods (PCEND, St. Louis Fed), divided by the total population (POP, St. Louis Fed). The risky return is the S\&P 500 return including dividend payments, and the risk-free return is the 1-month risk-free rate series from Professor Fama located in the CRSP data base. All series are deflated by the consumer price index for all urban consumers (CPIAUCSL, St. Louis Fed). Since both consumption series start in January 1959 in the St. Louis Fed data base, the data set ranges from January 1959 to December 2009.

The estimated time discount factor is 1.001 , with standard error equal to 0.001 and, therefore, it is not possible to reject the hypothesis $\beta_{0}<1$. The estimate of the elasticity of intertemporal substitution is 0.39 , with standard error 0.05 . Hence, it is significantly different from zero. The downside risk aversion is 0.43 , with standard error equal to 0.02 , being significantly different from 0.5 . Such results

\footnotetext{
${ }^{17}$ That is, from $S$ bootstrapped samples one estimates $S$ pairs $(\widehat{\lambda}, \widehat{\psi})$ and computes their empirical variance matrix.
} 
are qualitatively the same as those obtained under simulation (the time discount factor used in the simulation exercise was 0.9998 , the EIS was 0.6 and the downside risk aversion was 0.45 ).

\section{Conclusion}

We considered a framework where a single agent makes his decision about consumption-investment looking at worst-case scenarios, which depend on his degree of pessimism. This agent can be motivated by a well-known quote among professional investors: "Focus on the downside, and the upside will take care of itself".

Using the quantile utility maximizer agent of Manski (1988) and Rostek (2010), we attached the agent's degree of pessimism to a well defined parameter. As a consequence, we disentangled attitude towards risk and attitude towards intertemporal substitution in a novel way.

Two important results emerged. First, with only 3 preference-related parameters, the model was able to reproduce the historical averages and volatilities of the excess return, risk-free rare and consumption growth, the low covariance between stock return and consumption growth, the countercyclicality of the risk premium, and the procyclicality of the risk-free rate. Second, it was possible to estimate the EIS from an Euler equation in which such a parameter was separably identified. Related to the second result, a novel and simple two-step estimation procedure for the EIS was proposed.

The developed model was restricted to a single risky asset and a risk-free security. This was enough to address the proposed questions. From the present discussion, it is not clear how one could extend the model to allow for $n>1$ risky assets in order to study the cross-section of the returns. This is an interesting topic for future research.

A pure quantile maximizer agent is probably not a good representation for general behavior towards risk. Given that, the present model should be understood as a stylized and parsimonious study within the class of models that use asymmetric preferences over good and bad outcomes (as in prospect 
theory and disappointment aversion). As such, this study makes two important contribution to the literature. Given its ability to explain the financial puzzles parsimoniously, it (i) offers a simpler view regarding the relationship between asymmetric preferences and financial data, and (ii) provides evidence that the good empirical results obtained by the studies employing asymmetric preferences are not due to over-fitting. 


\section{Appendix}

\section{Proof of Proposition 1:}

Substituting the restrictions into the object function, the problem is given by

$$
\underset{\xi \in \mathbb{R}}{\operatorname{Max}} Q_{t}^{\tau}\left(u\left(W_{t}-P_{t} \xi-P_{t}^{f} \xi^{f}\right)+\beta u\left(X_{t+1} \xi+X_{t+1}^{f} \xi^{f}\right)\right)
$$

By the quantile equivariance, this is equivalent to

$$
\underset{\xi \in \mathbb{R}}{\operatorname{Max}} u\left(W_{t}-P_{t} \xi-P_{t}^{f} \xi^{f}\right)+\beta u\left(\xi Q_{t}^{\tau}\left(X_{t+1}\right)+X_{t+1}^{f} \xi^{f}\right)
$$

and the first order conditions are

$$
\begin{gathered}
\xi \quad: \quad u^{\prime}\left(C_{t}\right) P_{t}=\beta u^{\prime}\left(Q_{t}^{\tau}\left(C_{t+1}\right)\right) Q_{t}^{\tau}\left(X_{t+1}\right) \\
\xi^{f}: \quad u^{\prime}\left(C_{t}\right) P_{t}^{f}=\beta u^{\prime}\left(Q_{t}^{\tau}\left(C_{t+1}\right)\right) X_{t+1}^{f}
\end{gathered}
$$

which implies

$$
\begin{aligned}
P_{t} & =\beta \frac{u^{\prime}\left(Q_{t}^{\tau}\left(C_{t+1}\right)\right)}{u^{\prime}\left(C_{t}\right)} Q_{t}^{\tau}\left(X_{t+1}\right) \\
P_{t}^{f} & =\beta \frac{u^{\prime}\left(Q_{t}^{\tau}\left(C_{t+1}\right)\right)}{u^{\prime}\left(C_{t}\right)} X_{t+1}^{f}
\end{aligned}
$$

Specializing $u(c)=\frac{c^{1-\gamma}-1}{1-\gamma}$,

$$
\begin{aligned}
P_{t} & =\beta\left(Q_{t}^{\tau}\left(\frac{C_{t+1}}{C_{t}}\right)\right)^{-\gamma} Q_{t}^{\tau}\left(X_{t+1}\right) \\
P_{t}^{f} & =\beta\left(Q_{t}^{\tau}\left(\frac{C_{t+1}}{C_{t}}\right)\right)^{-\gamma} X_{t+1}^{f}
\end{aligned}
$$

\section{CQFD.}

\section{Proof of Proposition 2:}

The risky asset and risk-free asset prices are given, respectively, by

$$
\begin{aligned}
P_{t} & =\eta_{t} Q_{t}^{\tau}\left(X_{t+1}\right) \\
P_{t}^{f} & =\eta_{t} X_{t+1}^{f}
\end{aligned}
$$


where $\eta_{t} \equiv \beta\left(Q_{t}^{\tau}\left(\frac{C_{t+1}}{C_{t}}\right)\right)^{-\gamma}$.

An arbitrage opportunity occurs if and only if it is possible to construct $\Xi_{t}=\left(\xi_{t}, \xi_{t}^{f}\right)$ such that

$$
\begin{aligned}
\xi_{t} P_{t}+\xi_{t}^{f} P_{t}^{f} & =0 \\
\xi_{t} X_{t+1}+\xi_{t}^{f} X_{t+1}^{f} & \geq 0
\end{aligned}
$$

with the second equation holding as an inequality for at least one point in the support of $X_{t+1}$.

Substituting 24 into the first equation of 25 ,

$$
\begin{aligned}
& \xi_{t} \eta_{t} Q_{t}^{\tau}\left(X_{t+1}\right)+\xi_{t}^{f} \eta_{t} X_{t+1}^{f}=0 \\
\Rightarrow & \xi_{t}^{f} X_{t+1}^{f}=-\xi_{t} Q_{t}^{\tau}\left(X_{t+1}\right)
\end{aligned}
$$

which, into the second equation of 25 gives the necessary and sufficient condition for arbitrage,

$$
\xi_{t}\left(X_{t+1}-Q_{t}^{\tau}\left(X_{t+1}\right)\right) \geq 0
$$

with inequality for at least one point in the support of $X_{t+1}$.

Therefore, all we need to rule out arbitrage is to impose

$$
Q_{t}^{\tau}\left(X_{t+1}\right) \in\left(\min \left\{\operatorname{supp}\left(X_{t+1}\right)\right\}, \max \left\{\operatorname{supp}\left(X_{t+1}\right)\right\}\right)
$$

If $X_{t+1}$ is a continuous random variable, this is implied by imposing $\tau \in(0,1)$, CQFD.

\section{Proof of Proposition 3:}

First, note that if $\ln (x) \sim N\left(\mu, \sigma^{2}\right)$ then $Q^{\tau}(x)=\exp \left(\mu+\sigma \Phi^{-1}(\tau)\right)$. This holds since

$$
\begin{aligned}
& F_{X}(x)=\Phi\left(\frac{\ln x-\mu}{\sigma}\right) \\
\Rightarrow \quad F_{X}^{-1}(\tau) & =\exp \left(\mu+\sigma \Phi^{-1}(\tau)\right)
\end{aligned}
$$


According to 6,

$$
\begin{aligned}
\log \left(C_{t+1} / C_{t}\right) \mid t & \sim N\left(\mu_{c}, \sigma_{c}^{2}\right) \\
\log \left(R_{t+1}\right) \mid t & \sim N\left(\mu_{r}, \sigma_{r}^{2}\right)
\end{aligned}
$$

Therefore,

$$
\begin{aligned}
Q_{t}^{\tau}\left(C_{t+1} / C_{t}\right) & =\exp \left(\mu_{c}+\sigma_{c} \Phi^{-1}(\tau)\right) \\
Q_{t}^{\tau}\left(R_{t+1}\right) & =\exp \left(\mu_{r}+\sigma_{r} \Phi^{-1}(\tau)\right)
\end{aligned}
$$

Dividing both sides of 4 and 5 by $P_{t}$, and using the quantile equivariance property,

$$
\begin{aligned}
& 1=\beta\left(Q_{t}^{\tau}\left(\frac{C_{t+1}}{C_{t}}\right)\right)^{-\gamma} Q_{t}^{\tau}\left(R_{t+1}\right) \\
& 1=\beta\left(Q_{t}^{\tau}\left(\frac{C_{t+1}}{C_{t}}\right)\right)^{-\gamma} R_{t+1}^{f}
\end{aligned}
$$

where $R_{t+1}=\frac{X_{t+1}}{P_{t}}$.

Substituting 26 into 27 and taking logs from both sides,

$$
\log (\beta)-\gamma \mu_{c}-\gamma \sigma_{c} \Phi^{-1}(\tau)+\mu_{r}+\sigma_{r} \Phi^{-1}(\tau)=0
$$

Hence, since $E_{t}\left(r_{t+1}\right)=\mu_{r}$,

$$
E_{t}\left(r_{t+1}\right)=-\log (\beta)+\gamma \mu_{c}+\Phi^{-1}(\tau)\left(\gamma \sigma_{c}-\sigma_{r}\right)
$$

For the risk-free rate, using 28 and 26 in the same way,

$$
r_{t+1}^{f}=-\log (\beta)+\gamma \mu_{c}+\Phi^{-1}(\tau) \gamma \sigma_{c}
$$

Therefore,

$$
E_{t}\left(r_{t+1}-r_{t+1}^{f}\right)=-\sigma_{r} \Phi^{-1}(\tau)
$$




\section{CQFD.}

\section{Proof of Proposition 4:}

As in the proof of Proposition 3, we use the fact that if $\ln (x) \sim N\left(\mu, \sigma^{2}\right)$ then $Q^{\tau}(x)=$ $\exp \left(\mu+\sigma \Phi^{-1}(\tau)\right)$. Given that,

$$
\begin{aligned}
Q_{t}^{\tau}\left(C_{t+1} / C_{t}\right) & =\exp \left(\mu_{c}+\sigma_{t} \Phi^{-1}(\tau)\right) \\
Q_{t}^{\tau}\left(R_{t+1}\right) & =\exp \left(\mu_{r}+\varphi \sigma_{t} \Phi^{-1}(\tau)\right)
\end{aligned}
$$

Hence, using 27,

$$
\ln \beta-\gamma \mu_{c}-\gamma \sigma_{t} \Phi^{-1}(\tau)+\mu_{r}+\varphi \sigma_{t} \Phi^{-1}(\tau)=0
$$

and, since $E_{t}\left(r_{t+1}\right)=\mu_{r}$, we have

$$
E_{t}\left(r_{t+1}\right)=-\ln \beta+\gamma \mu_{c}+(\gamma-\varphi) \sigma_{t} \Phi^{-1}(\tau)
$$

For the risk-free rate, using 28 and the conditional quantile for consumption growth,

$$
r_{t+1}^{f}=-\ln \beta+\gamma \mu_{c}+\gamma \sigma_{t} \Phi^{-1}(\tau)
$$

Therefore,

$$
E_{t}\left(r_{t+1}-r_{t+1}^{f}\right)=-\varphi \sigma_{t} \Phi^{-1}(\tau)
$$

\section{CQFD.}

Proof of Proposition 5:

$$
\begin{aligned}
& E\left[\left(\tau_{0}-1\left[C_{t+1} / C_{t}<\left(\beta_{0} R_{t+1}^{f}\right)^{\psi_{0}}\right]\right) Z_{t} \mid \mathcal{F}_{t}\right] \\
= & \left(\tau_{0}-E\left[1\left[C_{t+1} / C_{t}<\left(\beta_{0} R_{t+1}^{f}\right)^{\psi_{0}}\right] \mid \mathcal{F}_{t}\right]\right) Z_{t} \\
= & \left(\tau_{0}-\operatorname{Pr}\left(\varepsilon_{c, t+1}<0 \mid \mathcal{F}_{t}\right)\right) Z_{t} \\
= & 0, \text { since } Q^{\tau_{0}}\left(\varepsilon_{c, t+1} \mid \mathcal{F}_{t}\right)=0 .
\end{aligned}
$$


Using the same steps, we also get

$$
E\left[\left(\tau_{0}-1\left[R_{t+1}<R_{t+1}^{f}\right]\right) Z_{t} \mid \mathcal{F}_{t}\right]=0 .
$$

CQFD. 


\section{References}

Bansal, R., and Yaron, A. (2004). "Risks for the long run: a potential resolution of asset pricing puzzles." Journal of Finance 59 (4): 1481-1509.

Bansal, R., Khatchatrian, V., and Yaron, A. (2005). "Interpretable asset markets?" European Economic Review 49 (3): 531-560.

Bansal, R., Kiku, D., and Yaron, A. (2009). "An empirical evaluation of the long-run risks model for asset prices.", working paper

Barberis, N., Huang, M., and Santos, T. (2001). "Prospect theory and asset prices." The Quarterly Journal of Economics, 116 (1): 1-53.

Bekaert, G., Hodrick, R., and Marshall, D. (1997). "The implications of first-order risk aversion for asset market risk premiums", Journal of Monetary Economics 40: 3-39.

Bonomo, M., and Garcia, R. (1993). "Disappointment aversion as a solution to the equity premium and the risk-free rate puzzles." Working paper, Université de Montréal.

Cooper, I., and Priestley, R. (2009). "Time-varying risk premiums and the output gap." Review of Financial Studies, 22(7): 2801-2833.

Engelhardt, G., and Kumar, A. (2009). "The elasticity of intertemporal substitution: new evidence from 401(k) participation". Economics Letters, 103(1): 15-17.

Epstein, L., and Zin, S. (1989). "Substitution, risk aversion, and the temporal behavior of consumption and asset returns: a theoretical framework". Econometrica, 57: 937-969.

- (1990). "First-order risk aversion and the equity premium puzzle", Journal of Monetary Economics, 26: 387-407. 
- (2001). "The independence axiom and asset returns", Journal of Empirical Finance 8: $537-572$.

Fama, F., and French, K. (1989). "Business conditions and expected returns on stocks and bonds." Journal of Financial Economics, 25, 23-49.

Feunou, B, Jahan-Parvar, M., and Tédongap, R. (2011). "Modeling Market Downside Volatility." Review of Finance, forthcoming.

Hansen, L., and Singleton, K.(1982). "Generalized instrumental variables estimation of nonlinear rational expectations models." Econometrica, 50, 1269-1286.

- (1983). "Stochastic consumption, risk aversion and the temporal behavior of asset returns." Journal of Political Economy, 91, 249-268.

Ludvigson, S., and Ng, S. (2009). "Macro factors in bond risk premia." Review of Financial Studies, 22(12): 5027-5067.

Manski, C. (1988). "Ordinal utility models of decision making under uncertainty." Theory and Decision, 25: 79-104.

Mehra, R., and Prescott,E. C. (1985). "The equity premium: a puzzle." Journal of Monetary Economics, 15: 145-61.

Rostek, M. (2010). "Quantile maximization in decision theory." Review of Economic Studies, $77(1): 339-371$

Routledge, B., and Zin, S. (2010). "Generalized disappointment aversion and asset prices." The Journal of Finance, forthcoming. 
Vissing-Jorgensen, A. (2002). "Limited asset market participation and the elasticity of intertemporal substitution." Journal of Political Economy, 110(4): 825-853.

Watcher, J. (2002). "Comment on: are behavioral asset-pricing models structural?" Journal of Monetary Economics, 49(1): 229-233.

Weil, P. (1989). "The equity premium puzzle and the risk-free rate puzzle." Journal of Monetary Economics, 24: 401-421.

Zin, S. (2002). "Are behavioral asset-pricing models structural?" Journal of Monetary Economics, 49(1): 215-228. 